\title{
Coded Bi-directional Relaying
}

\author{
Peter Larsson, Niklas Johansson, Kai-Erik Sunell \\ Ericsson Research
}

\begin{abstract}
In this summary, we consider bi-directional communication between a transmitter and receiver communicating via a relay station. We present a novel communication method based on joint data packet encoding in conjunction with exploitation of a priori known information that enables reduced number of transmissions, whereby enhancing aggregate throughput.

\section{Introduction}

Relaying schemes, e.g. manifested by multihopping [1] and cooperative relaying [2], is a hot research area for future wireless networks. In particular, relaying is envisioned as a possible method for enhancing today's cellular systems for extended range for high data rate services. A basic research challenge is to design more efficient relay schemes than existing methods. In this summary, we focus on the bidirectional traffic case and present a novel relaying method.
\end{abstract}

\section{Coded Bi-directional Relaying}

In a cellular system, traffic is communicated in both uplink and downlink between a Basestation (BS) and Mobile Station (MS). When introducing an intermediate relay station (RS), the simplest extension to the notion of downlink and uplink, could be to use four orthogonal resources for $\mathrm{BS} \rightarrow \mathrm{RS}, \mathrm{RS} \rightarrow \mathrm{MS}, \mathrm{MS} \rightarrow \mathrm{RS}$, and $\mathrm{RS} \rightarrow \mathrm{BS}$ transmissions, (henceforth denoted classical relaying). This is shown in Fig. 1 (left) with a four-phase protocol. We now propose a more efficient relay solution. The guiding observations towards the proposed method are:

- the wireless medium is of broadcast type.

- the bi-directional topology itself.

The core idea is to jointly encode BS and MS originating data in the relay, and when each node, i.e. BS and MS, receives the jointly encoded data, each exploits a priori information of its originally transmitted (and stored) data.

The basic idea is shown in Fig 1 (Right). In phase a) and b) transmission takes place from $\mathrm{BS} \rightarrow \mathrm{RS}$ and $\mathrm{MS} \rightarrow \mathrm{RS}$, and the relay decodes the data packets $\mathrm{D}_{1}$ and $\mathrm{D}_{2}$ respectively. In phase $\mathrm{c}$ ), the relay jointly encodes the data packets $\mathrm{D}_{1}$ and $\mathrm{D}_{2}$ with an XOR bitwise operation into a common data packet $\mathrm{D}_{3}$, i.e. prior to modulation and FEC encoding. Subsequent the RS multicast $\mathrm{D}_{3}$ to both the BS and the MS. At decoding at the BS, a bitwise XOR operation of the data packet $\mathrm{D}_{3}$ (after error correction and demodulation) and the a priori information $\mathrm{D}_{1}$ is performed, that the yields $\mathrm{D}_{2}$. The MS performs the corresponding operation. If the data packets are of unequal length, zero-padding of the shorter data sequence is used. When the link quality differs, the relay transmit power is preferably set according to the most stringent link requirement.

Several extensions of this basic scheme are envisioned., e.g. the idea of bi-directional 2-hop relaying is trivially extended to multiple hops. In addition, the direct signals
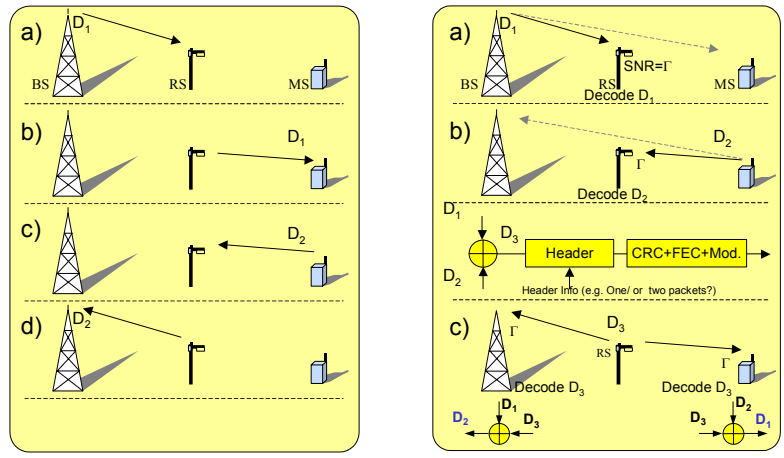

Fig. 1. Classical (left) and proposed (right) scheme

$\mathrm{BS} \rightarrow \mathrm{MS}$ and $\mathrm{MS} \rightarrow \mathrm{BS}$ can be exploited, e.g. through Incremental Redundancy and Chase combining strategies.

\section{Performance}

The proposed scheme's advantage stems from that only one relay transmission is needed, instead of two, yet without any extra power expenditure. As a very simple example, in the summary, we consider the aggregate throughput as performance measure with the following assumptions; identical path loss exist between BS-RS and MS-RS, the same transmit power level is used by all nodes, same amount of traffic originate from the BS and the MS, and direct BS-MS communication is neglected for simplicity reasons. Two cases are compared, i) classical four-phase relaying, ii) and the proposed three-phase relaying scheme. Under those assumptions, the classical case consumes exactly $4 / 3$ more resources than the proposed scheme at identical SNRs. Equivalently, the throughput gain is also a factor $4 / 3$. The gain is lower bounded to unit value when the path losses differ significantly. The throughput gain in bidirectional multihopping (with large number of hops) can be shown to be upper limited to a factor 2 .

\section{Discussion and Conclusions}

In this summary, a new communication scheme for bidirectional relaying has been proposed that removes the need of forwarding data to the BS and the MS in two different transmissions, but instead uses only one transmission. The gain for a simple symmetric path loss scenario was exactly $4 / 3$. Some extensions of the core idea have also been highlighted. ${ }^{1}$

\section{References}

[1] M. Frodigh, P. Johansson and P. Larsson, "Wireless ad hoc networking - The art of networking without a network", Ericsson Review No 4, 2000, p 248 - 257.

[2] A Sendonaris, "Advanced Techniques for NextGeneration Wireless Systems", Ph.D. Thesis, Rice University, August 1999

\footnotetext{
${ }^{1}$ Presented at the 5 th Scandinavian WS on Wireless Ad-hoc Networks (AdHoc'05), Stockholm, Sweden, May 2005.
} 